The cause of the headache is uncertain, but probably related to the tonsillar herniation. Intermittent obstructive hydrocephalus, with increased intracranial pressure, irritation of meninges at the foramen magnum, compression of upper cervical roots, and involvement of the descending nucleus of the trigeminal nerve are some of the possible causes of pain with Chiari-I malformation. (Van den Bergh R, Van Calenbergh F. Headache and headache-attacks in the Chiari-I malformation and in syringomyelia. Headache Quarterly 1997;8:15-21). (Reprints: Prof Dr Raymond Van den Bergh, Heidebergstraat 248 a B-3010 Leuven, Belgium).

COMMENT. A herniation of $5 \mathrm{~mm}$ is considered pathological and $3-5 \mathrm{~mm}$ is borderline. Minimal degrees of tonsillar herniation discovered by MRI in children with migraine type headaches is of concern and requires follow-up. Chiari-I headache may present at puberty or during first pregnancies. Hormonal changes may accentuate the tonsillar herniation and precipitate symptoms. (Progress in Pediatric Neurology II, PNB Publ, 1994;pp158-9).

\title{
INTRACRANIAL HYPERTENSION
}

\section{PSEUDOTUMOR CEREBRI AFTER L-THYROXINE THERAPY}

Pseudotumor cerebri in an infant treated with L-thyroxine for transient neonatal hypothyroidism is reported from the Montefiore Medical Center, Albert Einstein College of Medicine, Bronx, NY. The mother had Graves disease, treated with radioiodine ablation and thyroxine replacement. At 25 hours of life the baby was jittery and had tachycardia and respiratory distress. Thyroid function tests revealed thyrotoxicosis. Treatment with propylthiouracil was followed within 48 hours by clinical improvement, but after 3 weeks she became hypothyroid, possibly as a result of placental transfer of maternal thyroid-stimulating hormone receptor-blocking antibodies. After treatment with L-thyroxine, $10-15 \mathrm{mcg} / \mathrm{kg}$, at age 5 months she developed split sutures, a bulging fontanel, and a rapid increase in head circumference. The CT and normal CSF and electrolyte studies were compatible with pseudotumor cerebri. She recovered spontaneously with no change in the therapy. The thyroxine was discontinued at 1 year without relapse, and thyroid function, growth, head circumference, and motor development were normal. (Raghavan S, DiMartino-Nardi J, Saenger P, Linder B. Pseudotumor cerebri in an infant after L-thyroxine therapy for transient neonatal hypothyroidism. L Pediatr March 1997;130:478-480). (Reprints: Barbara Linder, MD, PhD, Division of Pediatric Endocrinology, Montefiore Medical Center, 111 East 210th Street, Bronx, NY 10467).

COMMENT. Previous reports of pseudotumor cerebri during the initiation of L-thyroxine therapy have involved children entering puberty, and hormonal mechanisms have been invoked. Apparently, this complication may also occur in infants and may require a slight modification of the dose of thyroxine.

\section{INTRACRANIAL HYPERTENSION AND CEREBRAL MALARIA}

Intracranial pressure (ICP) was monitored and cerebral perfusion pressure (CPP) calculated in 23 African children suffering from cerebral malaria and treated at the Kenya Medical Research Institute, Clinical Research Center, Kilifi, Kenya. Of 4 children with severe intracranial hypertension (ICP 
$>40 \mathrm{~mm} \mathrm{Hg}$, CPP $<40 \mathrm{~mm} \mathrm{HG),} 2$ died with transtentorial herniation and cardiorespiratory arrest, 2 had severe neurological sequelae. The remainder with mild ( $<20 \mathrm{~mm} \mathrm{Hg}$ ) or intermediate intracranial hypertension $(<40 \mathrm{~mm} \mathrm{Hg})$ survived without severe sequelae. Mannitol was of value only in children with intermediate intracranial hypertension and ICP $<40 \mathrm{~mm} \mathrm{Hg}$. Corticosteroids are detrimental in adults with cerebral malaria and have not been used in children. Osmotherapy is not recommended by the WHO. (Newton CRJC, Crawley $\mathrm{J}$, Sowumni A et al. Intracranial hypertension in Africans with cerebral malaria. Arch Dis Child March 1997;76:219-226). (Respond: Dr CRJC Newton, Neurosciences Unit, The Wolfson Centre, Mecklenburgh Square, London WC1N 2AP, UK).

COMMENT. The authors cite possible causes of intracranial hypertension in cerebral malaria, including increase in cerebral blood volume (CBV), cerebral edema, and hydrocephalus. Increased CBV seemed most likely from CT and MRI studies. Clinical signs of ICP, pupil dilatation and decerebrate posturing, are unreliable in estimating severity of intracranial hypertension, and the benefits of ICP monitoring need controlled evaluation. My colleague, Dr Charles Swisher, who treated malaria in the Service in Vietnam, referred to the interference with the vascular supply to the central nervous system in cerebral malaria, due to sludging and thrombosis caused by intravascular parasites, and consequent ischemia and anoxemia. Hyperpyrexia and convulsions are common symptoms. (Hunter GW III, Frye WW, Swartzwelder JC. A Manual of Tropical Medicine, 3rd ed, Philadelphia, WB Saunders, 1961).

\section{SEIZURES AND RELATED DISORDERS}

\section{IMMUNIZATION-INDUCED SYNCOPAL ATTACKS}

The clinical features and morbidity of a total of 697 cases of syncope after vaccination reported to the national Vaccine Adverse Reporting System were analyzed at the Center for Biologics Evaluation and Research, Food and Drug Administration, Rockville, Md. The vaccines implicated included measlesmumps-rubella (33\%), oral polio (15\%), tetanus-diphtheria, adult (15\%), D.T.P. $(12 \%)$, hepatitis B $(10 \%)$, influenza $(4 \%), \mathrm{H}$ influenza B (3\%), D.T., pediatric (2\%), D.T. acellular pertussis (2\%), and typhoid (1\%). Of 511 syncopes occurring within 1 hour of vaccination, $63 \%$ were within $5 \mathrm{~min}$ and $98 \%$ within $30 \mathrm{~min}$. Approximately $25 \%$ of attacks were complicated by tonic or clonic movements, and $10 \%$ of the total required hospitalization. Head injuries with skull fracture, intracranial bleeding, or cerebral contusion resulted from syncope-induced falls in 6 individuals, 12 to 28 years of age, and 3 required surgery, with neurologic deficits in 2. (Braun MM, Patriarca PA, Ellenberg SS. Syncope after immunization. Arch Pediatr Adolesc Med March 1997;151:255259). (Respond: M Miles Braun MD, HFM-220, Food and Drug Administration, 1401 Rockville Pike, Rockville, MD 20852).

COMMENT. Vaccinators should advise patients to sit for at least 15 minutes after immunization, and particularly following MMP vaccine. Those complaining of light-headedness should be checked for hypotension and bradycardia. A $25 \%$ incidence of convulsive movements reported in association with these post-immunization vasovagal attacks is of interest. Seizures in association with syncope following blood donation are not uncommon, and are rarely found to represent an epileptic event. Auras associated with syncope have some similarity to those preceeding epileptic seizures, but in contrast to epilepsy, syncopal auras lack localizing significance and may be less complex. 